\title{
ESTUDO DE CONFIGURAÇÕES PARA TROCADORES DE CALOR A PLACAS
}

\author{
L. F. NOVAZZI ${ }^{1}$ e T. V. MOURA ${ }^{1}$ \\ ${ }^{1}$ Centro Universitário da FEI, Departamento de Engenharia Química \\ E-mail para contato: lnovazzi@fei.edu.br
}

\begin{abstract}
RESUMO - Os trocadores de calor a placas são equipamentos flexíveis, com boa relação área de troca térmica / volume, proporcionam altos coeficientes de transferência de calor e apresentam facilidade para limpeza. Essa flexibilidade também se deve a diferentes possibilidades de configurações de escoamento, através de mudanças nas placas e gaxetas do trocador. Nesse trabalho modelou-se matematicamente o comportamento de um trocador de calor a placas, em regime permanente. Esse modelo foi obtido através de balanços de energia nos canais do trocador, por meio do método das diferenças finitas, resultando num sistema de equações lineares. A validação experimental do modelo foi feita num trocador a placas de pequena escala, de laboratório. Foram estudadas diferentes configurações para os trocadores, e para cada uma delas se fez uma análise dos fatores de correção das médias logarítmicas de temperatura. Mostrou-se que esse fator é fortemente dependente das configurações em jogo.
\end{abstract}

\section{INTRODUÇÃO}

Em indústrias de processos químicos, é bastante comum o uso de trocadores de calor casco e tubo para promover aquecimento ou resfriamento de correntes (Kern, 1987). Contudo, vem crescendo bastante o emprego de trocadores de calor a placas (PHEs), que proporcionam elevadas taxas de transferência de calor e podem operar com baixa diferença de temperatura entre as correntes. De modo simplificado, os PHEs são constituídos por placas metálicas corrugadas, paralelas entre si e entre as quais escoam os fluidos através dos canais formados, com vazão orientada por gaxetas (Shires et al., 1994). O arranjo de um PHE é representado por $P^{I} \times N^{I} / P^{I I} \times N^{I I}$, sendo $P$ o número de passes e $N$ o número de passagens, enquanto que os algarismos romanos sobrescritos $I$ e $I I$ indicam os dois diferentes lados do trocador nas placas. Na Figura 1 (Gut, 2003) são indicadas algumas possibilidades de arranjo para um PHE de nove placas e oito canais de escoamento. Na representação do canto superior esquerdo, tem-se um arranjo paralelo tipo U, com apenas um passe dos fluidos no trocador e quatro passagens, o que é indicado pela notação $1 \times 4$ / 1×4. Já no arranjo mostrado no canto superior direito, o arranjo é paralelo, mas numa configuração tipo Z. No canto inferior esquerdo tem-se um arranjo em série, numa representação dada por $4 \times 1 / 4 \times 1$. No canto inferior direito representa-se um arranjo mais complexo, dado por $2 \times 2 / 1 \times 4$. Cada diferente arranjo influencia diretamente na troca térmica, fazendo com que ela seja maior ou menor no PHE. 


\section{9 a 22 de outubro de 2014 \\ Florianópolis/SC}

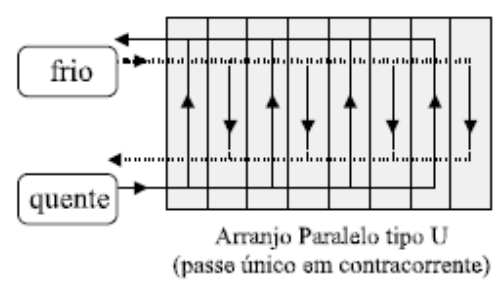

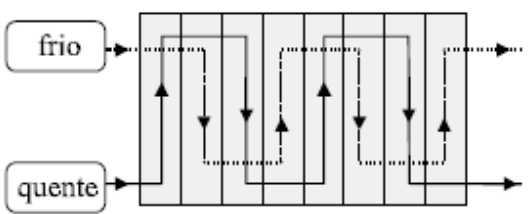

Arranjo em Série ( 4 passes de 1 canal / 4 passes de 1 canal)

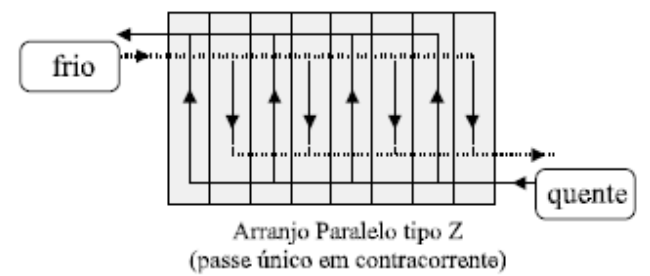

Arranjo Paralelo tipo Z (passe único em contracorrente)

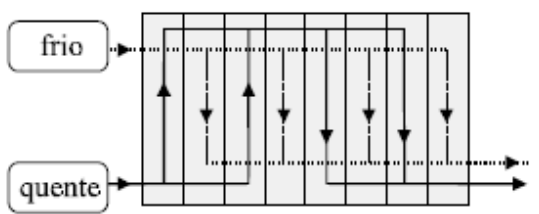

Arranjo Complexo ( 2 passes de 2 canais 1 passes de 4 canais)

Figura 1 - Quatro configurações num PHE.

Os métodos de dimensionamento rigorosos de PHEs não são disponíveis em literatura aberta, sendo propriedade dos fabricantes desses equipamentos. Além disso, esse dimensionamento é dependente da configuração utilizada no PHE, que pode ser bastante variada. No trabalho de Gut (2003) empregaram-se técnicas de otimização para determinação das melhores configurações de operação para os PHEs, em regime estacionário. O modelo matemático desenvolvido por esse autor foi posteriormente validado através da comparação com resultados experimentais de um PHE de placas lisas.

No trabalho de Gutierrez et al. (2011) fez-se um estudo da distribuição dos tempos de residência (RTD) num PHE de placas lisas, usado para pasteurização, com configurações de escoamento em série e em paralelo. Esses autores observaram através dos dados experimentais que o modelo de dispersão axial para a RTD apresenta bons resultados para um número maior de passes no PHE, em virtude da turbulência nos canais do equipamento.

O uso de fluidodinâmica computacional (CFD) em PHEs também pode ser encontrado na literatura. No trabalho de Galeazzo et al. (2006), foi modelado através de CFD um PHE de quatro canais, com placas lisas. Os autores analisaram configurações de escoamento em série e em paralelo e também compararam os resultados previstos por CFD, no qual se consideram todos os efeitos em três dimensões, com um modelo mais simplificado, representado por fluxo pistonado. Os resultados obtidos por CFD foram próximos ao do modelo mais simplificado, com desvios de $8 \%$ e boa concordância com os dados experimentais. Entretanto, os autores mostraram que o uso de CFD é mais vantajoso por gerar como resultado um perfil detalhado das temperaturas e das velocidades nos canais no PHE.

Além do comportamento térmico, também pode ser encontrada na literatura uma avaliação de perda de carga num PHE (Miura et al., 2008). Nessa pesquisa, os autores analisaram a influência de 32 diferentes configurações sobre a perda de carga em PHEs, usando os resultados experimentais para determinar uma correlação empírica para o efeito do número de passes e de canais sobre a variação de 


\section{9 a 22 de outubro de 2014 \\ Florianópolis/SC}

pressão no equipamento. Esses pesquisadores também validaram um fator de atrito para o PHE e posteriormente trataram do problema da perda de carga usando CFD. No contexto apresentado nessa revisão bibliográfica, o objetivo principal desse trabalho envolve uma análise de como as diferentes configurações de um PHE afetam na troca térmica e no fator de correção da média logarítmica de temperaturas. Como objetivos específicos, avalia-se experimentalmente os coeficientes convectivos e o coeficiente global de transferência de calor num PHE de laboratório, validando-se o modelo matemático proposto através de comparação com os resultados experimentais.

\section{METODOLOGIA}

\subsection{Experimental}

Foi utilizado nesse projeto um PHE modelo HT32, de área total de 0,04 $\mathrm{m}^{2}$ e 5 placas de troca térmica, fabricado pela Armfield. Emprega-se como fluido de troca água quente e água fria. A água quente opera em circuito fechado, com sistema automático de controle de temperaturas. Já a água fria é disponível da rede, sem nenhum controle adicional. Existem quatro termopares no trocador, do tipo $\mathrm{K}$, que medem as temperaturas de entrada e de saída dos fluidos. Também há dois medidores de vazão volumétrica, do tipo turbina. As principais características do trocador são apresentadas na Tabela 1, sendo $F_{T}$ o fator de correção da média logarítmica de temperaturas.

Tabela 1 - Características do PHE

\begin{tabular}{ccc}
\hline Parâmetro & Unidade & Valor \\
\hline Área da placa & $\mathrm{m}^{2}$ & $8 \cdot 10^{-3}$ \\
Espessura da placa & $\mathrm{m}$ & $5 \cdot 10^{-4}$ \\
Condutividade das placas & $\mathrm{W} \cdot \mathrm{m}^{-1} \cdot \mathrm{K}^{-1}$ & 15 \\
Área dos canais & $\mathrm{m}^{2}$ & $1,15 \cdot 10^{-4}$ \\
Diâmetro hidráulico & $\mathrm{m}$ & $3 \cdot 10^{-3}$ \\
$F_{T}$ & & 0,90 \\
Número de placas & & 5 \\
\hline
\end{tabular}

Para a aquisição dos dados experimentais, o PHE foi montado com um total de 6 canais, num arranjo em série $3 \times 1 / 3 \times 1$, semelhante àquele apresentado no canto inferior esquerdo da Figura 1, mas com o fluido quente e frio entrando por baixo do trocador. Foi ajustado um valor de referência para a temperatura de entrada do fluido quente, $T_{H, i n}$, igual a $50{ }^{\circ} \mathrm{C}$, enquanto que a temperatura de entrada do fluido frio, $T_{C, i n}$, foi igual à temperatura disponível na rede. As vazões volumétricas de água quente e fria $\left(F_{H}\right.$ e $\left.F_{C}\right)$ ensaiadas foram de $1,00 \mathrm{~L} \cdot \mathrm{min}^{-1}$ até $2,50 \mathrm{~L} \cdot \mathrm{min}^{-1}$, com variações de 0,25 em $0,25 \mathrm{~L} \cdot \mathrm{min}^{-1}$ entre cada combinação testada. Para as diferentes combinações de vazão, esperaramse 30 minutos de operação no trocador, a fim de garantir que o regime permanente tivesse sido atingido. Depois disso, os dados foram amostrados com frequência de $10 \mathrm{em} 10 \mathrm{~s}$, até se obter 25 pontos para cada combinação de vazão especificada. Foram registradas as temperaturas de entrada e de saída dos fluidos e suas respectivas vazões volumétricas. Dos 25 pontos de cada ensaio, foram escolhidos os cinco melhores, que proporcionavam um menor erro entre a carga térmica trocada entre 


\section{9 a 22 de outubro de 2014 \\ Florianópolis/SC}

as correntes quentes e frias. Tendo-se em conta a pequena influência da temperatura sobre o calor específico da água no intervalo de temperaturas estudado, considerou-se um $C_{P}$ constante de 4180 $\mathrm{J} \cdot \mathrm{kg}^{-1} \cdot \mathrm{K}^{-1}$. Contudo, a variação da densidade da água com a temperatura foi levada em conta nos resultados.

\subsection{Modelagem Matemática}

Na parte de modelagem matemática, o comportamento térmico do PHE é dado pela Equação 1, na qual $Q$ é a carga térmica, $U$ é o coeficiente global de transferência de calor, $A$ é a área de troca térmica, $\Delta T_{L M}$ é média logarítmica de temperaturas e $F_{T}$ é o fator de correção da média logarítmica de temperaturas (Kakaç e Liu, 2000).

$$
Q=U A \Delta T_{L M} F_{T}
$$

A carga térmica do trocador é determinada experimentalmente, por meio do balanço de energia das correntes. A média logarítmica de temperaturas também é determinada experimentalmente, através dos valores das temperaturas de entrada e de saída das correntes. A área de troca térmica é conhecida e o fator de correção da média logarítmica foi especificado pelo fabricante na configuração empregada. Desse modo, através da Equação 1, determina-se o coeficiente global de troca térmica $U$ do trocador. Para um trocador sem incrustações, esse coeficiente é uma função das resistências convectivas dos fluidos e condutiva da placa, como escrito na Equação 2, na qual $h_{I}$ e $h_{I I}$ são os coeficientes convectivos dos fluidos nos dois lados dos canais, $e$ é a espessura da placa e $k$ é sua condutividade térmica.

$$
U=\frac{1}{\frac{1}{h_{I}}+\frac{1}{h_{I I}}+\frac{e}{k}}
$$

Os coeficientes convectivos são uma função das características de escoamento e de propriedades termodinâmicas e de transporte dos fluidos. Como se trata de um trocador água / água, se forem mantidas as mesmas condições de vazão para a corrente quente e a fria, os coeficientes $h_{I} \mathrm{e}$ $h_{I I}$ serão próximos entre si, já que se trata de uma geometria semelhante no escoamento. Uma das propostas desse trabalho é avaliar esses coeficientes, através de uma correlação como aquela indicada na Equação 3. Nessa equação, $N u$ é o número de Nusselt, $R e$ o número de Reynolds e $\operatorname{Pr}$ o número de Prandtl. Os coeficientes $a_{1}, a_{2}$ e $a_{3}$ são ajustados por meio dos resultados experimentais, e nesse trabalho admitiu-se que $a_{3}$ seria igual a $1 / 3$, valor esse encontrado em muitas correlações apresentadas em literatura.

$$
N u=a_{1} \operatorname{Re}^{a_{2}} \operatorname{Pr}^{a_{3}}
$$

A modelagem da troca térmica nos canais é baseada no esquema apresentado na Figura 2, para placas de largura $w$, altura $L$ e largura dos canais igual a $b$. São consideradas as seguintes hipóteses no modelo: regime permanente, perdas de calor desprezíveis, sem mudança de estado, fluxo pistonado de escoamento e coeficiente global de troca térmica constante. Faz-se um balanço de energia tomando-se como volume de controle o $i$-ésimo canal, numa distância que vai de $x$ até $(x+\delta x)$. Se $\delta x$ for 
suficientemente pequeno, pode-se assumir que a temperatura $T_{i}$ seja constante nesse trecho do canal, caracterizando mistura perfeita.

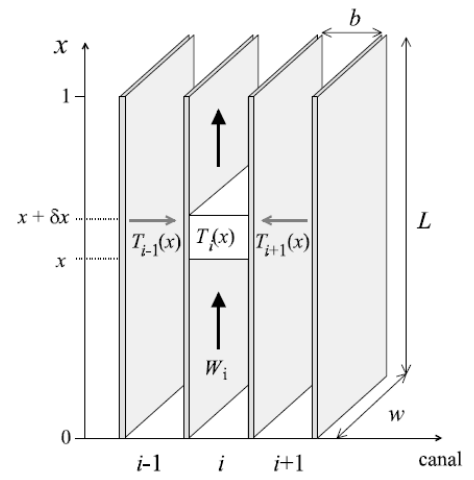

Figura 2 - Modelo de canais num PHE.

Fonte: Gut, 2003

Esse balanço resulta na Equação 4 , na qual $W_{i}$ representa a vazão mássica no canal $i, C_{p, i}$ é o calor específico da corrente, $\partial A_{p}$ é uma área infinitesimal de troca térmica e $T_{i-1}(x)$ e $T_{i+1}(x)$ são as temperaturas dos fluidos nos canais adjacentes $(i-1)$ e $(i+1)$, respectivamente. Nesse trabalho a Equação 4 foi implementada para diferentes arranjos num PHE. Como essa equação é escrita para cada trecho discretizado do trocador, tem-se como resultado um sistema linear de equações para ser resolvido, o que foi feito em Matlab. Essa discretização consiste numa aplicação do método das diferenças finitas (Constantinides e Mostoufi, 1999).

$$
W_{i} C_{p, i}\left[T_{i}(x)-T_{i}(x+\delta x)\right]+\partial A_{p} U\left[T_{i-1}(x)-T_{i}(x)\right]+\partial A_{p} U\left[T_{i+1}(x)-T_{i}(x)\right]=0
$$

$\mathrm{Na}$ Figura 3 é apresentado um esquema do arranjo em série $3 \times 1 / 3 \times 1$ do PHE de laboratório e que foi utilizado na discretização, com cinco placas de troca de calor e seis canais. As linhas vermelha e azul indicam o fluido quente e frio, respectivamente.

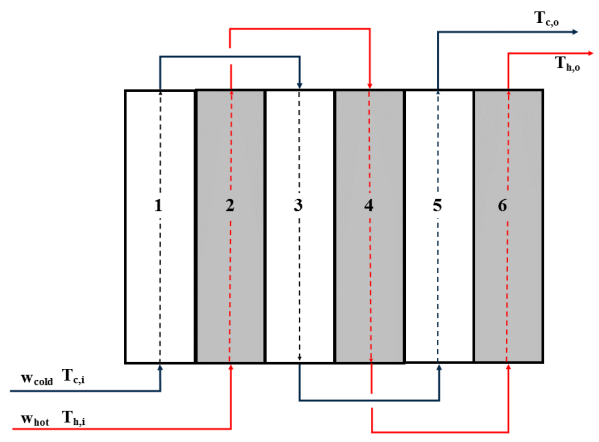

Figura 3 - Configuração de escoamento do PHE. 


\section{RESULTADOS E DISCUSSÃO}

$\mathrm{Na}$ Tabela 2 são apresentados os resultados experimentais obtidos com o PHE. Esses valores representam as médias dos cinco melhores pontos obtidos e o coeficiente global experimental $U_{\text {exp }}$ foi calculado através da Equação 1.

Tabela 2 - Resultados experimentais

\begin{tabular}{cccccccc}
\hline $\begin{array}{c}F_{H} / \\
\left(\mathrm{L} \cdot \text { min }^{-1}\right)\end{array}$ & $\begin{array}{c}F_{C} / \\
\left(\mathrm{L} \cdot \min ^{-1}\right)\end{array}$ & $T_{H, \text { in }} /{ }^{\circ} \mathrm{C}$ & $T_{H, \text { out }} /{ }^{\circ} \mathrm{C}$ & $T_{C, \text { in }} /{ }^{\circ} \mathrm{C}$ & $T_{C, \text { out }} /{ }^{\circ} \mathrm{C}$ & $Q / \mathrm{W}$ & $\begin{array}{c}U_{\text {exp }} / \\
\mathrm{W} \cdot \mathrm{m}^{-2} \cdot \mathrm{K}^{-1}\end{array}$ \\
\hline 1,05 & 0,99 & 49,3 & 39,0 & 24,0 & 35,4 & 753 & 1451 \\
1,21 & 1,26 & 49,8 & 39,2 & 24,4 & 35,1 & 886 & 1673 \\
1,52 & 1,49 & 49,4 & 39,3 & 23,9 & 34,2 & 1056 & 1924 \\
1,77 & 1,74 & 49,5 & 39,4 & 23,9 & 34,6 & 1226 & 2243 \\
2,00 & 1,99 & 49,6 & 39,5 & 24,0 & 34,2 & 1385 & 2491 \\
2,30 & 2,24 & 48,4 & 39,3 & 24,0 & 33,3 & 1452 & 2649 \\
2,49 & 2,49 & 46,4 & 38,1 & 23,8 & 32,1 & 1429 & 2773 \\
\hline
\end{tabular}

A partir das Equações 2 e 3 e desde que $a_{1}$ e $a_{2}$ sejam especificados, é possível estimar um valor para o coeficiente global do trocador, $U_{c a l c}$. Através do método dos mínimos quadrados, foram ajustados os coeficientes $a_{1}$ e $a_{2}$ da Equação 3, de modo a minimizar os desvios quadráticos entre $U_{\text {calc }}$ e $U_{\text {exp }}$. O ajuste foi bastante razoável, conforme se demonstra na Figura 4. Os valores encontrados de $a_{1}$ e $a_{2}$ foram respectivamente iguais a 0,052 e 0,796 .

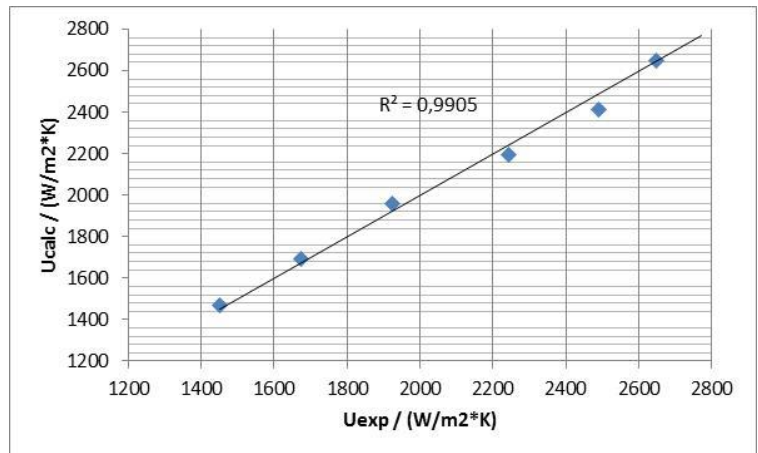

Figura 4 - Comparação entre o coeficiente global estimado e o experimental.

A fim de se avaliar a modelagem matemática apresentada na seção 2.2, a Equação 4 foi aplicada para os canais discretizados do PHE, de arranjo em série $3 \times 1 / 3 \times 1$. Cada canal foi discretizado em 100 intervalos diferentes, resultando num sistema linear com 600 variáveis. Na Figura 5 são indicadas as comparações para as temperaturas das correntes de saída entre os resultados previstos pelo modelo e aqueles obtidos experimentalmente. Pode-se ver que a aproximação do modelo foi boa, com elevado coeficiente de correlação, tanto para a corrente quente como para a corrente fria. 


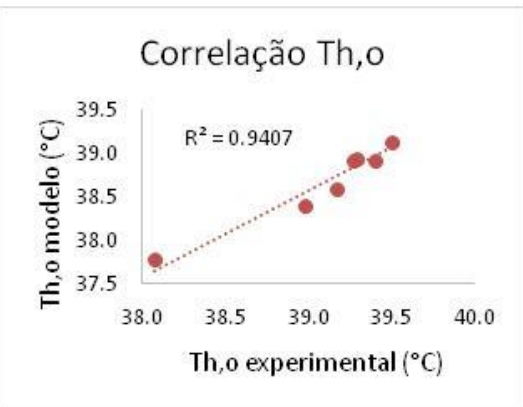

(a) Fluido quente

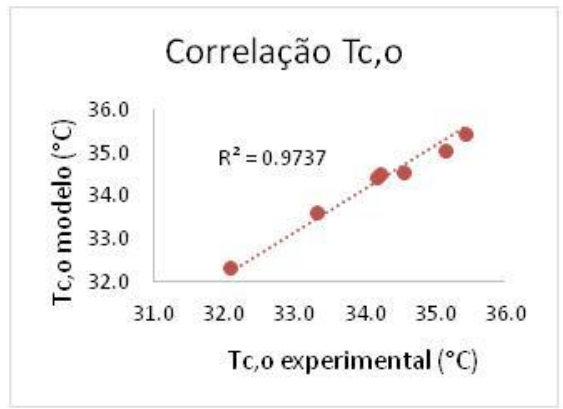

(b) Fluido frio

Figura 5 - Comparação do modelo discretizado do trocador com resultados experimentais

Na Figura 6 são apresentados os valores dos fatores de correção das médias logarítmicas de temperaturas, $F_{T}$, como função de duas diferentes configurações e como função dos adimensionais $R$ e $S$, respectivamente definidos como $\frac{T_{H, \text { in }}-T_{H, \text { out }}}{T_{C, \text { out }}-T_{C, \text { in }}}$ e $\frac{T_{C, \text { out }}-T_{C, \text { in }}}{T_{H, \text { in }}-T_{C, \text { in }}}$.

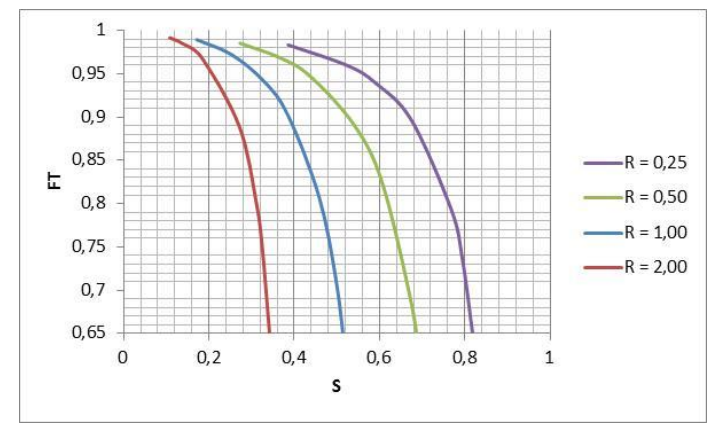

(a) Arranjo em série $2 \times 1 / 2 \times 1$.

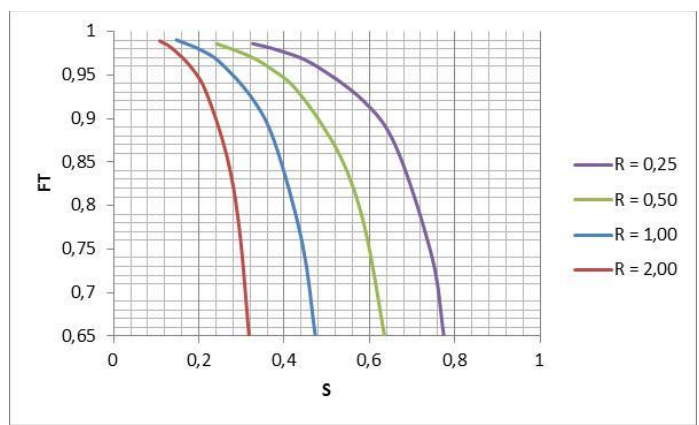

(b) Arranjo em série $4 \times 1 / 4 \times 1$.

Figura 6 - Fator de correção da média logarítmica de temperaturas.

Os coeficientes globais experimentais exibidos na Tabela 2 foram calculados a partir de um fator $F_{T}$ igual a 0,90 (Tabela 1). Contudo, esse fator de correção é influenciado pela configuração de escoamento e também pelos adimensionais $R$ e $S$. Na Tabela 3 são apresentados os fatores $F_{T}$, calculados a partir do modelo discretizado, para as diferentes condições ensaiadas em laboratório. Nota-se que a hipótese de se assumir um fator de correção igual a 0,90 é razoável.

Tabela 3 - Fator de correção da média logarítmica para os ensaios

\begin{tabular}{ccccc}
\hline$F_{H} /\left(\mathrm{L} \cdot \mathrm{min}^{-1}\right)$ & $F_{C} /\left(\mathrm{L} \cdot \mathrm{min}^{-1}\right)$ & $R$ & $S$ & $F_{T}$ \\
\hline 1,05 & 0,99 & 0,91 & 0,45 & 0,84 \\
1,21 & 1,26 & 0,99 & 0,42 & 0,85 \\
1,52 & 1,49 & 0,98 & 0,40 & 0,87 \\
1,77 & 1,74 & 0,95 & 0,42 & 0,87 \\
2,00 & 1,99 & 0,99 & 0,40 & 0,87 \\
2,30 & 2,24 & 0,98 & 0,38 & 0,89 \\
2,49 & 2,49 & 1,00 & 0,37 & 0,90 \\
\hline
\end{tabular}




\section{9 a 22 de outubro de 2014 \\ Florianópolis/SC}

\section{CONCLUSÕES}

O dimensionamento de trocadores de calor do tipo PHE apresenta relação de dependência direta da configuração utilizada. Para estudar essa relação, foi analisado um esquema em série $3 \times 1 / 3 \times 1$, com o objetivo de desenvolver um modelo matemático para avaliar condições de operação do trocador. A partir dos dados experimentais de um PHE, foi ajustada uma correlação para a estimativa do coeficiente global de transferência de calor do equipamento como função das condições de escoamento e de propriedades do fluido (Equação 3), obtendo-se boa aproximação (Figura 4). O PHE foi modelado em regime permanente, através de balanços de energia nos canais do trocador, por meio do método das diferenças finitas. Verificou-se que o modelo representou bem o comportamento das temperaturas das correntes no trocador (Figura 5). A partir desse modelo, foi determinado o fator de correção das médias logarítmicas de temperaturas para duas diferentes configurações num PHE (Figura 6). Mostrou-se que esse fator é dependente das configurações em jogo.

\section{REFERÊNCIAS}

CONSTANTINIDES, A., MOSTOUFI, N. Numerical Methods for Chemical Engineers with Matlab Applications. Nova Iorque: Editora Prentice Hall, 1999.

GALEAZZO, F.C.C.; MIURA, R.Y.; GUT, J.A.W.; TADINI, C.C. Experimental and Numerical Heat Transfer in a Plate Heat Exchanger. Chemical Engineering Science, v.61, n.21, p.7133-7138, 2006.

GUT, J.A.W. Configurações Ótimas para Trocadores de Calor a Placas. Tese (doutorado). São Paulo: Universidade de São Paulo, Escola Politécnica, 2003.

GUTIERREZ, C.G.C.C.; DIAS, E.F.T.S.; GUT, J.A.W. Investigation of the Residence Time Distribution in a Plate Heat Exchanger with Series and Parallel Arrangements using non-ideal Tracer Detection Technique. Applied Thermal Engineering, v.31, n.10, p.1725-1733, 2011.

KAKAÇ, S.; LIU, H. Heat Exchangers: Selection, Rating and Thermal Design, 2 ed. Boca Raton: Editora CRC Press, 2000.

KERN, D. Processos de Transmissão de Calor. Rio de Janeiro: Editora Guanabara Koogan, 1987.

MIURA, R.Y.; GALEAZZO, F.C.C.; TADINI, C.C.; GUT, J.A.W. The Effect of Flow Arrangement on the Pressure Drop of Plate Heat Exchangers. Chemical Engineering Science, v.63, n.22, p.5386-5393, 2008.

SHAH, R.K.; FOCKE, W.W. Plate Heat Exchangers and their Design Theory. In: SHAH, R.K.; SUBBARAO, E.C.; MASHELKAR, R.A. (Ed.) Heat Transfer Equipament Design. Hemisphere P.C., 1988, p.227-254.

SHIRES, G.L.; BOTT, T.R.; HEWITT, G.F. Process Heat Transfer. Londres: Editora CRC Press, 1994. 